Narjara de Lemos Barbosa ${ }^{1}$

Suzana de Oliveira Mangueira ${ }^{1}$

Jaqueline Galdino Albuquerque ${ }^{1}$

Fernanda Jorge Guimarães ${ }^{1}$

\author{
Nursing care for alcohol- \\ ic patient: perceptions \\ team of the nursing
}

\begin{abstract}
Introduction: The alcohol abuse is a relevant issue of public bealth and it is related to a variety of problems such as physical, psychic and social. Objective: This study aims to analyze a nurse staff perception on factors affecting alcoholic pacient care. Methods: A descriptive, exploratory qualitative approach conducted in the city of Vitoria de Santo Antão/PE, Brazil. The data were colected using semi-structured interviews and content analysis. Results: The nurse care of alcoholic patient is driven by a discriminatory behavior from the nurse staff, even though they understand alcoholism as a chronic disease that need to be treated. Conclusion: This study contributes to advance nursing practices by providing subsidies about the relation alcoholic patient - nurse professional.
\end{abstract}

Keywords | Nursing; Nursing team; Nursing care; Alcoholism.

\section{| Cuidado de Enfermagem a pacientes alcoolistas: percepçóes da equipe de enfermagem}

RESUMO| Introdução: O abuso de álcool está relacionado com diversos problemas, físicos, psíquicos, sociais, o que o torna um relevante problema de saúde pública. Objetivo: $\mathrm{O}$ estudo teve como objetivo conhecer a percepção da equipe de Enfermagem sobre o cuidado a pacientes alcoolistas. Métodos: Estudo descritivo, exploratório com abordagem qualitativa, realizado no município da Vitória de Santo Antão/PE. Os dados foram coletados por entrevistas com a utilização de um roteiro semiestruturado e avaliados a partir da técnica de análise de conteúdo. Resultados: Constatou-se que o cuidado de Enfermagem a pacientes alcoolistas está permeado por uma relação estigmatizada dos profissionais com esses pacientes, mesmo com a percepção do alcoolismo como uma doença crônica que necessita ser tratada. Conclusão: O estudo contribui para o avanço da Enfermagem, pois fornece subsídios para que se conheça o cuidado prestado pela equipe de Enfermagem aos pacientes usuários de álcool.

Palavras-chave | Enfermagem; Equipe de enfermagem; Cuidados de Enfermagem; Alcoolismo. 


\section{INTRODUÇÃO|}

O álcool é uma droga psicotrópica que tem seu uso admitido e incentivado pela sociedade. Quanto às suas implicações, o álcool exerce efeito depressor sobre o sistema nervoso central (SNC), cuja gravidade depende de sua concentração na corrente sanguínea.

Entre os efeitos causados pelo consumo de bebidas alcoólicas, destacam-se o aumento do risco para problemas sociais, de trabalho, familiares, físicos e legais, o que o configura como problema de saúde pública ${ }^{1}$. Observase crescimento do consumo de álcool paralelamente ao aumento dos problemas sociais, associado ao estigma e ao preconceito sofrido pelos alcoolistas, que são vistos, na maioria das vezes, como pessoas indesejáveis, inconvenientes, desmoralizadas e indisciplinadas ${ }^{2}$.

Dados do II Levantamento Domiciliar sobre o Uso de Drogas Psicotrópicas no Brasil, realizado nas 108 maiores cidades do País, revelam que 74,6\% da população total fazem ou fizeram uso de álcool alguma vez na vida e em 12,3\% há prevalência de dependência ${ }^{3}$. Diante disso, observa-se um elevado consumo de álcool e uma prevalência significativa de dependência na população brasileira.

Dessa forma, a equipe de Enfermagem mostra-se como um dos principais prestadores de assistência aos alcoolistas, pois, por meio de seu processo de trabalho, encontra-se mais próxima dessa clientela. Apesar dessa realidade e da existência de pesquisas no Brasil sobre a assistência de Enfermagem a indivíduos com problemas relacionados com o consumo excessivo de álcool, identificaram-se dois estudos direcionados aos enfermeiros ${ }^{4,5}$, em detrimento dos demais membros da equipe de Enfermagem.

Assim, ao considerar a problemática do abuso de álcool e a importância da equipe de Enfermagem nesse atendimento, emergiu a necessidade da realização deste estudo, que tem como objetivo conhecer a percepção da equipe de Enfermagem sobre o cuidado a pacientes alcoolistas.

\section{MÉTODOS|}

Trata-se de um estudo descritivo, exploratório, com abordagem qualitativa. A pesquisa foi realizada em um hospital filantrópico conveniado à rede do Sistema Único de Saúde (SUS). A escolha da instituição justificouse pelo fato de se tratar de uma unidade de referência, com maior capacidade de atendimento a indivíduos com complicações crônicas decorrentes do abuso do álcool.
O hospital foi inaugurado em 1953, com a criação de um Posto de Puericultura. Em 1955, passou a incorporar serviço de maternidade e, desde 2002, realiza atendimento a agravos e doenças crônicas causadas pelo álcool, na unidade de cuidados prolongados.

Atualmente, o hospital conta com 123 leitos distribuídos nas clínicas de Pediatria, Obstetrícia, Clínica Médica, Cirurgia Geral, Cirurgia Ginecológica, Cirurgia Vascular, Traumato - Ortopedia e Unidade de Cuidados Prolongados. Possui oito enfermarias para o atendimento de pacientes com síndrome da dependência alcoólica, distribuídas em duas alas, que perfazem 39 leitos.

O Serviço de Atenção e Reabilitação ao Portador de Síndrome da Dependência Alcoólica, realizado na unidade de cuidados prolongados, engloba: internação de pacientes alcoolistas, intervenção interdisciplinar e multiprofissional, desenvolvimento da espiritualidade, resgate da cidadania com o restabelecimento do vínculo familiar, encaminhamento e acompanhamento pós-alta hospitalar para a unidade de referência em alcoolismo, intervenção psicoterapêutica no processo de internação, parceria com os Alcoólicos Anônimos (AA), grupo de acolhimento, palestras educativas, grupos de autoajuda para desenvolvimento da autoestima, grupo de avaliação do tratamento, grupo de preparação para a alta hospitalar, oficinas de arte, atividades de lazer e recreação, atividade física, parceria com outras instituições as quais desenvolvem assistência aos usuários de drogas para estabelecimento do sistema de referência e contrarreferência.

Em sua maioria, os usuários são do sexo masculino, com faixa etária de 19 a 65 anos, oriundos do próprio município e de cidades circunvizinhas, além de pessoas provenientes da Capital, região metropolitana e outras cidades do agreste e sertão pernambucano. $\mathrm{O}$ serviço atende ainda à clientela indígena encaminhada pela Fundação Nacional do Índio (Funai). O tempo de permanência dos pacientes no serviço é variável e depende do quadro clínico deles. Em média, tem duração de 30 dias e pode estender-se até 90 dias. Após a alta hospitalar, o paciente é encaminhado para continuidade do tratamento em nível ambulatorial na rede pública que trata alcoolistas, e os residentes no município são encaminhados para o ambulatório do próprio hospital.

O hospital possui um quadro funcional de 217 profissionais, dos quais 57 são de nível superior, 160 de nível médio. $\mathrm{Na}$ unidade de cuidados prolongados, ficam cinco técnicos de Enfermagem e um enfermeiro por plantão. O setor não atende a casos de pacientes com intoxicação alcoólica ou outras situações emergenciais. O enfoque é na condição crônica. Entretanto, destaca-se que, nas primeiras semanas de internamento, é comum os 
pacientes apresentarem episódios agudos decorrentes da síndrome de abstinência. $\mathrm{O}$ atendimento aos alcoolistas é realizado por uma equipe multidisciplinar, a qual envolve: terapeuta ocupacional, fonoaudióloga, fisioterapeuta, psicóloga, nutricionista, assistente social e médico, além da equipe de Enfermagem, sujeitos do estudo.

Participaram do estudo sete profissionais da equipe de Enfermagem, escolhidos por meio da técnica "bola de neve" e que atendessem aos princípios de inclusão e exclusão. Os critérios de seleção dos sujeitos foram: período de contratação no serviço superior a 60 dias e participação diretamente da assistência a pacientes alcoolistas crônicos. O critério de exclusão foi encontrar-se afastado do serviço por licença médica, maternidade ou férias. Os participantes do estudo trabalham no setor que atende aos alcoolistas. Os critérios foram adotados para o alcance dos objetivos propostos no estudo. O número de sujeitos entrevistados foi definido com base no critério de saturação das respostas durante a coleta dos dados, ou seja, quando os dados novos trouxeram informações repetidas ${ }^{6}$.

A coleta de dados foi desenvolvida no período compreendido entre dezembro de 2011 e fevereiro de 2012, por meio de entrevistas gravadas, a partir de um roteiro semiestruturado que continha a questão norteadora, a saber: "1-Como você compreende o cuidado de enfermagem a pacientes alcoolistas?”. As entrevistas ocorreram após a assinatura do Termo de Consentimento Livre e Esclarecido (TCLE) pelos participantes.

O relato oral de cada profissional foi transcrito na íntegra e, após sua leitura exaustiva, o material empírico foi categorizado de acordo com a técnica de análise de conteúdo ${ }^{7}$. Tal análise desenvolveu-se em pré-análise, que é a fase de organização do material; exploração do material, caracterizada pela administração sistemática das decisões tomadas; e tratamento dos resultados, inferência e interpretação.

Dessa forma, a análise seguiu os seguintes passos: leitura flutuante das entrevistas, releituras e demarcações de expressões significativas das falas dos sujeitos que, em seguida, passaram por um processo de fragmentação, interpretação e compreensão. Foram agregadas conforme as expressões semelhantes que apresentaram uma ideia central, ou seja, uma categoria, e resultou, assim, na formação de categorias temáticas baseadas nas falas dos sujeitos.

O estudo foi aprovado pelo Comitê de Ética e Pesquisa do Centro de Ciências da Saúde da Universidade Federal de Pernambuco, sob protocolo $n^{\circ} 314 / 11$, em respeito aos aspectos éticos da pesquisa que envolve seres humanos, como preconizado pela Resolução no 196/96, do Conselho Nacional de Saúde.

\section{RESULTADOS |}

Os sujeitos que participaram do estudo eram, em sua maioria, do sexo feminino, com idade entre 28 e 50 anos, sendo duas enfermeiras e cinco técnicos em enfermagem. Três profissionais possuíam cursos de capacitação e dois tinham curso de pós-graduação nas áreas de conhecimento da Enfermagem.

A análise do material empírico permitiu identificar duas categorias temáticas relacionadas com a percepção dos profissionais de Enfermagem sobre o cuidado aos alcoolistas: a) percepção sobre o cuidado de Enfermagem a pacientes alcoolistas; e b) alcoolismo: uma doença crônica. Essas categorias descrevem as percepções dos profissionais sobre o alcoolismo.

\section{Percepção sobre o cuidado de Enfermagem a pacientes alcoolistas}

Com relação à primeira categoria temática, ao manifestarem sua percepção sobre os cuidados técnicos de Enfermagem a pacientes alcoolistas, foi imperativa nas falas dos profissionais a relação entre o cuidado e o seguimento das normas estabelecidas pela instituição e pelos profissionais da equipe.

[...] é mais difícil quando opaciente está em abstinência alcoólica. $\mathrm{Na}$ maioria das vezes, chega aqui desorientado, totalmente desorientado. Não sabe a importância do tratamento, não quer aceitar as medicações, fica agressivo. Então, isso atrapalha um pouco o cuidado de Enfermagem. Mas, quando passa a abstinência, o paciente fica orientado, colabora mais com as normas do hospital, são prestativos [...] (E.1).

[...] muitos deles, enquanto estão internos, respeitam as normas do hospital e dos profissionais que estão cuidando deles. Agora, quando eles estão fora do hospital, é totalmente diferente, porque eles ficam muito junto dos amigos, e os amigos influenciam a beber [...] quando estão em casa, não obedecem ninguém. Acham que todo mundo quer se meter na vida deles [...] (E.2).

[...] é mais paciência do que o próprio cuidado. Eles são pacientes difíceis. A maioria é paciente de rua. E essas pessoas de classe menos favorecida não têm uma educação tão boa. As vezes não obedece, não quer fazer as coisas. Então, é mais paciência [...] (E.3).

[...] no tratar com eles [...] têm uns que são difíceis. Eles são muito dificeis! Ultimamente tinha um aqui [...], meu Deus! 
Muito indisciplinado. Indisciplinado de verdade. Recebeu até alta por indisciplina e eu não sou de dizer nada de nenbum paciente, porque en adoro todos! Mas realmente ele dizia muitos palavrões, muitos mesmo. Descia da cadeira, se arrastava no chão [...] coisa difícil. Arrumando problema, confusão com os colegas, fumando dentro do banbeiro. Um absurdo [...] (E.5).

Diante das falas apresentadas, verificou-se que o cuidado de Enfermagem é restrito apenas aos procedimentos técnicos, e o não seguimento das normas da instituição atrapalha a prestação desse cuidado.

\section{Alcoolismo: uma doença crônica}

A partir dos discursos dos sujeitos, foi possível evidenciar suas ideias sobre o alcoolismo. Tais percepções são relevantes, pois podem influenciar o modo como é realizado o cuidado aos usuários de álcool.

Dessa forma, com relação à segunda categoria temática, quando expressam suas percepções sobre o alcoolismo, a análise das falas permitiu evidenciar que, em sua maioria, os profissionais da equipe de Enfermagem reconhecem o alcoolismo como doença crônica, o que pode ser observado nos discursos a seguir:

[...] o alcoolismo é uma doença crônica que não tem cura, $e$ sim um tratamento. Muitas vezes, os nossos pacientes passam um periodo de três a seis meses e, após a alta, na maioria das vežes, não são todos que seguem o tratamento, que reconbecem que realmente o alcoolismo é uma doença [...] (E.1).

[...] o alcoolismo é uma doença. Não é o fato de beber porque quer. É uma doença e é difícil de tratar [...] (E.3).

[...] eu vejo o alcoolismo como uma doença que começa sem você perceber... Porque um alcoolista começa feito eu ou você. A gente vai numa festinha e bebe socialmente, como a gente costuma dizer. E todo mundo que está aqui começou assim, mas, por algum motivo, começou a fazer o uso descontrolado do álcool [...] (E.7).

Constatou-se, também, no discurso de dois profissionais, que houve uma ampliação na percepção do alcoolismo, que é visto por eles como um problema que envolve aspectos biológicos, psicológicos e sociais:

[...] eu acho que é um problema familiar, a falta de emprego, a falta de renda, acho que contribui também para o alcoolismo. Falta de apoio familiar [...] eu acho que o consumo excessivo de álcool está principalmente ligado à parte financeira, falta de apoio familiar e falta de lazer para a população [...] (E.4).

[...] o alcoolismo é uma doença, mas, também, tem vários fatores por trás: tem a desestrutura familiar, o desemprego, a falta de oportunidades na vida, entre outras coisas [...] (E.6).

Diante desses resultados, pode-se afirmar que o alcoolismo é percebido pelos profissionais da equipe de Enfermagem como uma doença crônica, que envolve fatores biopsicossociais, sem cura, mas que possui tratamento.

\section{DISCUSSÃO|}

Os discursos dos participantes do estudo mostram que ainda há uma visão estereotipada sobre o alcoolista, a qual, na maioria das vezes, é influenciada pelo contexto social. Ao assumir essa relação com os alcoolistas, a sociedade tende a rotulá-los como pessoas ignorantes, criminosas, loucas... Tal estigma persiste ao longo dos séculos, de modo a influenciar atitudes e percepções dos profissionais responsáveis pelo cuidado a esses pacientes ${ }^{8}$.

Diante dos resultados apresentados, evidenciou-se que os participantes da pesquisa percebem os alcoolistas como pessoas difíceis de lidar, desobedientes, indisciplinadas e que não seguem as normas da instituição. A pesquisa descreveu as concepções de enfermeiros sobre os alcoolistas como pessoas difíceis de lidar, agressivas, que perturbam, tumultuam e atrapalham a dinâmica do serviço, o que pode tornar a prática mais difícil. Os enfermeiros possuem dificuldades para entender certos comportamentos do alcoolista, como manifestações de sintomas de doenças ou de intoxicação aguda? .

A percepção de cuidados de Enfermagem voltados apenas para procedimentos técnicos e seguimento de normas refere-se, geralmente, a uma abordagem tradicional e, há muito tempo, preconiza-se um cuidado individualizado aos pacientes. Cuidado este que não foi observado na análise das falas dos entrevistados.

Semelhante ao presente estudo, pesquisa com enfermeiros identificou que as ações voltadas aos alcoolistas são heterogêneas e fragmentadas de acordo com a visão de cada profissional. A ausência de ações voltadas às especificidades dessa clientela se dá pela falta de programas de atenção na unidade e à falta de preparo dos profissionais ${ }^{10}$.

O processo formativo do profissional de Enfermagem não aborda profundamente conteúdos sobre os cuidados aos 
usuários de álcool e de outras drogas. Pesquisa realizada com alunos do último período do curso de graduação em Enfermagem de instituições privadas apontou que as atitudes de estudantes de Enfermagem em frente ao alcoolista foram negativas, por considerá-lo culpado por seus problemas e preferem não trabalhar com esse usuário $^{11}$. Esse resultado mostra a fragilidade na formação dos futuros enfermeiros, o que pode afetar a qualidade da assistência de Enfermagem a pacientes usuários de álcool e outras drogas. Tal resultado mostra-se como ponto relevante na assistência de Enfermagem a pacientes usuários de álcool, já que tal percepção sobre o alcoolismo influencia a prestação de cuidados. Outro estudo sugere maior atenção ao treinamento dos enfermeiros para a abordagem ao alcoolista em hospital geral ${ }^{12}$.

Com relação à segunda categoria temática, que se refere à percepção sobre o alcoolismo, os profissionais entrevistados demonstraram que o alcoolismo é concebido como uma doença. Esse resultado soma-se com outro encontrado na literatura que evidencia concepção semelhante ${ }^{5}$. Corrobora um estudo que evidenciou que os enfermeiros se referem ao uso de álcool como doença, o cuidado é focado no tratamento e respaldado no modelo biomédico ${ }^{8}$.

O alcoolismo é, geralmente, definido como um conjunto de fenômenos comportamentais, cognitivos e fisiológicos que se desenvolve após o consumo consistente e excessivo tipicamente associado à preocupação em tomar a droga e a dificuldade de controlar o seu consumo, ao ponto de esse comportamento interferir na vida pessoal, social, familiar e/ou profissional do indivíduo ${ }^{13}$.

Estudo realizado com enfermeiros de Unidades de Atenção Básica Distritais de Saúde de um município paulista, com o objetivo de conhecer as concepções e as tendências de suas atitudes em frente ao álcool, alcoolismo e alcoolista, mostra que não houve distinção em relação ao presente estudo quanto à percepção do alcoolismo como doença. No entanto, entre os entrevistados, ainda emergiram falas que remeteram ao estigma que recai sobre o alcoolista ${ }^{5}$.

Corrobora um estudo que identificou que os profissionais percebem o alcoolismo como doença, destacando o álcool como a droga responsável. Esses profissionais possuem uma representação alicerçada em estereótipos e estigmas em relação ao paciente ${ }^{14}$.

Dessa forma, os resultados desta pesquisa condizem com os de pesquisas anteriores. Contudo, houve uma incoerência nas falas dos profissionais entrevistados, pois percebem o alcoolismo como doença, mas ainda com resquícios do modelo moral, em que sua etiologia é vista como fraqueza de caráter. Os profissionais da equipe de Enfermagem do estudo, embora sejam integrantes de uma instituição hospitalar de referência para o cuidado a pacientes alcoolistas crônicos e integrarem uma equipe multidisciplinar voltada para esse cuidado, demonstraram, em suas falas, lacunas em competências e habilidades para a prestação do cuidado a esses pacientes.

O trabalho multidisciplinar é uma importante ferramenta que os profissionais de Enfermagem podem utilizar para incrementar o cuidado ao paciente alcoolista. Estudo realizado com acadêmicos de Enfermagem apontou a saúde como uma área essencialmente interdisciplinar, que envolve cooperação, respeito à diversidade, colaboração e diálogo ${ }^{15}$. Nessa perspectiva, a equipe de Enfermagem pode adotar um cuidado integral, o qual valoriza aspectos psicossociais e técnicos em seu trabalho.

O entendimento do alcoolismo como doença crônica pelos profissionais é válido e contribui na prestação dos cuidados; no entanto, nas falas dos sujeitos do estudo, perceberamse fragilidades nessa contribuição, pois os alcoolistas são vistos de forma estigmatizada, ao mesmo tempo em que são considerados doentes que necessitam de tratamento.

\section{CONCLUSÃO|}

Verificou-se que o objetivo do estudo foi alcançado. Por meio da análise das entrevistas, foi possível observar a percepção da equipe de Enfermagem sobre o cuidado dispensado a pacientes alcoolistas como também sobre o alcoolismo. A análise do material empírico identificou um cuidado pautado no cumprimento de normas e rotinas. Evidenciou-se a percepção do alcoolismo como uma doença crônica que necessita ser tratada; no entanto, para se tratar com qualidade qualquer patologia, seja física, seja psíquica, os profissionais da equipe de Enfermagem necessitam articular os conhecimentos científicos com o cuidado humanizado.

Dessa maneira, sugere-se a necessidade de discussões, treinamentos e capacitações dos profissionais envolvidos no cuidado direto a pacientes alcoolistas, porquanto, como demonstrado neste estudo, há fragilidade dos conhecimentos teórico-práticos fornecidos a esses profissionais em sua formação.

Como limitação deste estudo, aponta-se a realização em único cenário de atuação do enfermeiro. Sugere-se o desenvolvimento de novas pesquisas em outros serviços que assistem a pacientes alcoolistas. 
A pesquisa possui relevância para a prática, pois fornece subsídios para que se conheça o cuidado prestado pela equipe de Enfermagem aos pacientes usuários de álcool, bem como suas limitações e potencialidades.

\section{REFERÊNCIAS |}

1 - Reinaldo AMS, Pillon SC. Repercussões do alcoolismo nas relações familiares: estudo de caso. Rev Latino-am Enfermagem [Internet]. 2008 mai/jun2008 [citado mai 2010 05]; 16(especial): [cerca de 6 p.]. Disponível em: http://www.eerp.usp.br/rlae

2 - Portugal FB, Corrêa APM, Siqueira MM. Alcoolismo e comorbidade em um programa de assistência aos dependentes de álcool. Rev Eletrônica Saúde Mental Alcool Drog. 2010; 6(1):1-13.

3 - Brasil. Secretaria Nacional Antidrogas. Gabinete de Segurança Institucional. II Levantamento domiciliar sobre o uso de drogas psicotrópicas no Brasil: estudo envolvendo as 108 maiores cidades do país - 2005. São Paulo: CEBRID/UNIFESP; 2006.

4 - Vargas D. Atitudes de enfermeiros de hospital geral frente às características pessoais do paciente alcoolista. Rev Bras Enferm. 2010; 63(6):1028-34.

5 - Vargas D, Luis MAV. Álcool, alcoolismo e alcoolista: concepções e atitudes de enfermeiros de unidades básicas distritais de saúde. Rev Latino-am Enfermagem [Internet]. $2008 \mathrm{mai} / j u n$ [citado 2011 nov 01]; 16(especial): [cerca de 8 p.]. Disponível em: http://www. eerp.usp.br/rlae

6 - Thiry Cherques HR. Saturação em pesquisa qualitativa: estimativa empírica de dimensionamento. Revista PMKP [Internet]. 2009 set [citado 2013 mai 01]; 3: [cerca 8 p.]. Disponível em: http://www.revistapmkt.com.br/ VOLUMESANTERIORES.aspx?udt_863_param_ detail $=113$.

7 - Bardin L. Análise de conteúdo. Lisboa: editora 70; 2011.

8 - Moretti-Pires RO, Ferro SBG, Büchele F, Oliveira HM de, Gonçalves MJF. Enfermeiro de Saúde da Família na Amazônia: conceitos e manejo na temática do uso de álcool. Rev Esc Enferm USP [Internet]. 2011 ago [citado 2012 jul 04]; 45(4):926-32. Disponível em: URL:http://www.scielo.br/scielo.php?script=sci_ arttext\&pid $=$ S008062342011000400019\&lng=en
9 - Vargas D, Oliveira MAF, Luis MAV. Atendimento ao alcoolista em serviços de atenção primária à saúde: percepções e condutas do enfermeiro. Acta Paul Enferm. 2010; 23(1):73-9.

10 - Souza IM, Pinto MG. Atuação do enfermeiro a usuários de álcool e de outras drogas na saúde da família. Rev Eletrônica Enferm [Internet]. 2012 abr/jun [citado 2013 jun 17];14(2):374-83. Disponível em: http://dx.doi. org/10.5216/ree.v14i2.11245

11 - Vargas D de. Atitudes de estudantes de enfermagem frente questões relacionadas ao álcool, alcoolismo e alcoolista. Acta PaulEnferm [Internet]. 2011 [citado2012 jul04];24(5):638-44. Disponível em: http:/ /www.scielo.br/scielo.php?script=sci_ arttext\&pid $=$ S010321002011000500007\&lng $=$ en

12 - Vargas D de. Atitudes de enfermeiros de hospital geral frente às características pessoais do paciente alcoolista. Rev Bras Enferm [Internet]. 2010 nov/dez [citado 2013 mai 5]; 63(6): 1028-34. Disponível em: http://www.scielo.br/scielo.php?script=sci_arttext\&pid $=$ S003471672010000600024

13 - Centro Brasileiro de Classificação de Doenças [Internet]. São Paulo: Faculdade de Saúde Pública da Universidade de São Paulo; c1993-2007 [citado 2011 out 30]. Manual da Classificação Estatística Internacional de Drogas e Problemas Relacionados à Saúde; [cerca de 360 telas]. Disponível em: http://www.datasus.gov.br/cid10/ V2008/cid10.htm

14 - Meira S, Arcoverde MAM. Representações sociais dos enfermeiros de unidades básicas de um distrito sanitário de Foz do Iguaçu, PR, sobre o alcoolismo. Rev Eletrônica Saúde Mental Alcool Drog [Internet]. 2010 [citado 2012 jul 5]; 6(1):1-13. Disponível em: http:/ / pepsic.bvsalud.org/scielo.php? script $=$ sci_ tte\&pid $=$ S180669762010000100012\&lng $=$ pt\&nrm $=$ iso $>$. ISSN 1806-6976.

15 - Oliveira ERA, Fiorin BH, Lopes LJ, Gomes MJ, Coelho SO, Morra JS. Interdisciplinaridade, trabalho em equipe e multiprofissionalismo: concepções de acadêmicos de enfermagem. Rev Bras Pesqui Saúde. 2011; 13(4):28-34. Responsável pela correspondência:

Fernanda Jorge Guimarães

Rua Manoel Pereira de Oliveira, 40

Cruz das Armas - João Pessoa - PB

E-mail:ferjorgui@hotmail.com

Recebido em: 14-12-2012

Aceito em: 27-6-2013 